\title{
Suffa Radio Observatory in Uzbekistan: progress and radio-seeing research plans
}

\author{
Alisher Hojaev ${ }^{1} \dagger$, G.I. Shanin ${ }^{2}$ and Yu.N. Artyomenko ${ }^{3}$ \\ ${ }^{1}$ Ulugh Beg Astronomical Institute, Center for Space Research, Uzbek Academy of Sciences, \\ Tashkent, Uzbekistan \\ email: ash@astrin.uzsci.net \\ ${ }^{2}$ Radio-observatory RT-70, Center for Space Research, Uzbek Academy of Sciences, Tashkent, \\ Uzbekistan \\ email: suffa@mail.ru \\ ${ }^{3}$ Astro-Space Center, Lebedev Physical Institute, Russian Academy of Sciences, Moscow, \\ Russia \\ email: feli@asc.rssi.ru
}

\begin{abstract}
The Suffa International Radio Observatory to be completed in coming years and the new radio astro-climate (seeing) research proposals for radio weather forecasting are described.
\end{abstract}

Keywords. Astronomy in Uzbekistan, telescopes, instrumentation: adaptive optics, radio continuum, lines: general, submillimetre, site testing, atmospheric effects

\section{Introduction}

Radio-astronomy is a rapidly developing and very promising branch of modern astrophysics. The SKA, ALMA, the space radio telescope and other prominent projects of coming years will open new horizons in the study of the universe in unexplored ranges and with much higher spatial resolution than in other spectral bands and with higher sensitivity than before.

One of the large-scale radio-astronomy facilities is the complex of the International Radio Astronomy Observatory being created on the Suffa plateau (Uzbekistan) in close collaboration with Russia (Kardashev, 1992). It should be a basic part of the Earth-Space VLBI system ( Kardashev et al., 1995). With the main instrument similar to the GBT at NRAO it will be one of the main basic elements of the global radio interferometry network as well. The Suffa project has been mentioned as one of the most prominent for the new century astronomy in the millennium overview paper (Trimble, 2001).

\section{General Information}

The location of the created observatory is shown in Fig. 1. The project itself was started up in 1984 and since 1992 project was suspended after the Soviet Union disappeared. In 1995 the agreement was signed between the governments of Russia and Uzbekistan establishing 'The International Radio Astronomy Observatory "Suffa"' (IRAOS). This agreement provides a legal basis for the continuation of the construction of the $70-\mathrm{m}$ Suffa radio-telescope which will belong to the IRAOS. The duration of the agreement is 99 years. The agreement is open for joining by other states, international organizations, national scientific institutions or personalities from third states.

There are at least three forms of joining to this project:

$\dagger$ Present address: UBAI, Astronomicheskaya 33, Tashkent 700052, UZB 


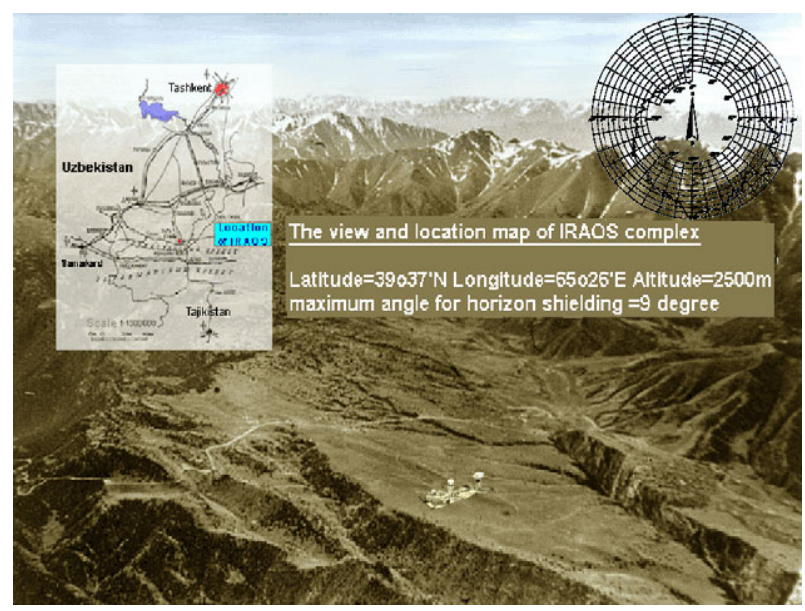

Figure 1. A mountain view of observatory on Suffa plateau. The location map and the telescope horizon shielding diagram are inserted in the left and top right, respectively.
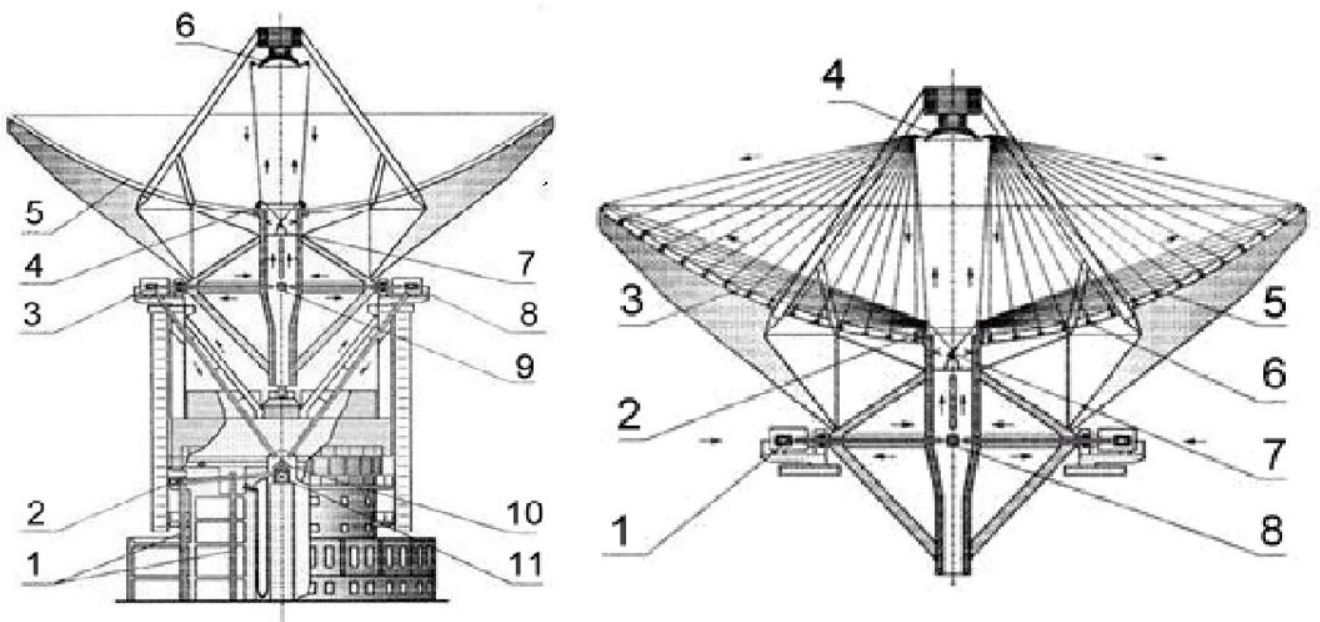

Figure 2. Sketch-section of RT-70 radio-telescope (left) and its primary mirror shape fitting system (right). Numbering in the left panel: 1 - azimuth master; 2, 3, 8 - laser position measuring devices; 4 - basic platform; 5 - primary mirror; 6 - counter-reflector; 7 - periscope mirror; 9 guide-builder(for precise pointing); 10 - monitoring device (controller); 11 - digital feedback sensor. Numbering in the right panel: 1 - laser position measuring devices; 2 - basic platform; 3 - primary mirror; 4 - counter-reflector; 5 - reference devices; 6 - electric jacks for reflecting panels; 7 - periscope mirror; 8 - guide-builder.

(a) the participation in completing the complex. An immediate participation in completing the installation is encouraged and will give the preferences in future activities;

(b) the participation in providing facilities by last generation receivers and other modern equipment. This might be started with participation in the new site testing project (see Chapter Radio Astro Climate. New Project of this report);

(c) scientific collaboration with co-financing of operation of the observatory.

Quite recently (2001) the firm decision on completing the project has been endorsed by our governments, and Russia will invest for these; therefore the project's layouts have been considerably modernized and updated in order to build up the state-of-art instrument. The telescope operation start time is planned for 2009. 

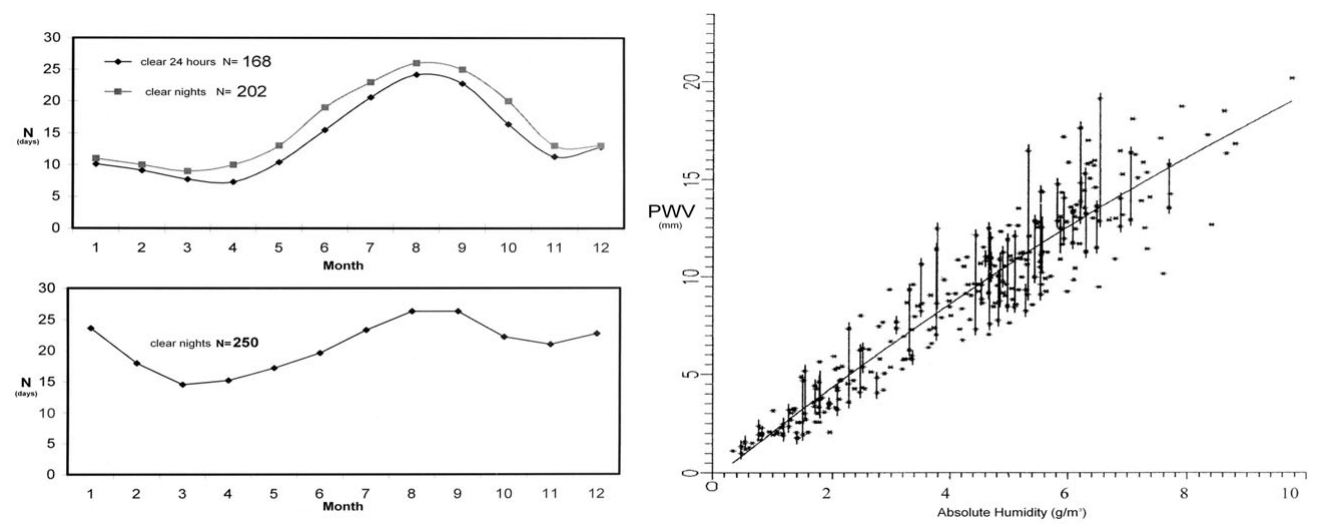

Figure 3. Left panel: distribution of clear time in 1981-1991 - general cloudiness (top left) and lower atmosphere cloudiness (bottom left). Right panel : correlation between absolute humidity and precipitable water vapour (PWV)

The purpose of the RT-70 radio-telescope should be in the following areas:

(a) Interferometry mode observations

(1.1) Earth-Space Very Long Base Interferometry(ESVLBI) with spaceborne radiotelescopes: (1.1.1) Radioastron $(D=10 \mathrm{~m})$, (1.1.2) Millimetron/Submillimetron $(D=12 \mathrm{~m})$ and (1.1.3) VSOP-2 at the angle resolution $10^{3}$ times better than ground-based VLBI existed;

(1.2) Ground-based VLBI within the existing networks; and

(b) Autonomous mode observations of the astrophysical objects and SETI targets. One should note there are no radio facilities or their creation projects in Central Asia and in its close vicinity, especially large ones, which makes the Suffa radio telescope an unique in the region. Thus and so the advantageous geographical location of the telescope will also make it indispensable for the global radio interferometry system.

Some main features of RT-70 radio-telescope are following. An optical arrangement is two-mirror Gregory scheme $(\mathrm{Feq}=571(345) \mathrm{m})$ with additional periscope mirror $D_{\text {per }}=$ $0.6 \mathrm{~m}$. In the left panel of Fig. 2 the sketch-section of the telescope is presented. The primary mirror is parabolic and has $D=70 \mathrm{~m}, F=21 \mathrm{~m}$, aperture angle $=160^{\circ}$. The secondary mirror(s) have ellipsoidal shape with $D=3(5) \mathrm{m}, I F=242 \mathrm{~m}$. The pointing accuracy is about 1 arc sec, which is achieved by using a special guide-builder and precise digital angle sensors. Guiding accuracy should be about 0.3 second of arc per second. Operational wavelengths are: in S-range $(0.87 \div 10 \mathrm{~mm})$ and in M-range $(1 \div 10 \mathrm{~cm})$ totally in the 7 (10) working bands. Total construction weight will be 4500 tonnes.

A compensation of the mirror system deformations caused by the weight and wind load, fluctuation in temperature, will be realized by homologous construction in longer wavelengths and by active adjustment of primary mirror shape in the mm-range (adaptive mirror - see Fig. 2, right panel). For this purpose special laser position measuring devices and individual electric jacks (in total 2000) will be used for each of 1188 reflecting panels (rms error $<50$ microns) of the prime mirror. The accuracy of the prime mirror profile after correction should be not less than 60 microns (Shanin, 1996). The directional pattern of the radio telescope in autonomous mode observations will be 3 " and the sensitivity in the mm-range up to $100 \mu \mathrm{J}$ which makes the facility unique. 


\section{Radio astro-climate}

\subsection{Previous site testing}

The site location provides good seeing conditions for $\mathrm{cm}-\mathrm{mm}$ range (see, e.g., Hojaev \& Shanin, 1996). Site tests started in 1981. The meteorological measurements have been carried out regularly since then. Sounding balloon and pilot-balloon measurements have also been made. The transparency in different mm bands has been estimated by using the radiometer measurements of atmospheric self-radiation. Averaged annual atmospheric transmission coefficients at the zenith were derived as $0.90 \div 0.98$ for $3.1 \mathrm{~mm}$ and $5.8 \mathrm{~mm}$ wavelengths and about 0.60 for $1.36 \mathrm{~mm}$, with a maximum in the winter time. The clear time estimations were also made. In Fig. 3 we give some results of testing the observing conditions at the Suffa site.

\subsection{New project}

Now we are arranging the scientific consortium in order further to explore the Suffa site more deeply and to learn the main 'radio astro-climate' parameters by means of a new technology ('radio-seeing', radio-transparency and stability in different sub-mm, $\mathrm{mm}$ and $\mathrm{cm}$ bands, PWV , their intercorrelation and correlation with meteo-parameters) for the atmosphere modelling at the site, and we try to forecast the "radio-weather" for reliably planning the scientific schedule of the future telescope. The proposals have been prepared to submit to the international funding agencies to support the project. To carry out these experiments we need radiometers especially $225 \mathrm{GHz}, 183 \mathrm{GHz}, 89 \mathrm{GHz}$ and automated meteorological stations. For this research any proposals and partners would be very desirable.

The $183.3 \mathrm{GHz}$ heterodyne receiver radiometer (see, e.g., Hills et al. 2001) centered on water molecule line which absorbs the space radiation in the appropriate $\mathrm{mm}$-band when it passes the atmosphere seems the best to probe for the water wapour content. Measuring at the three double-side frequency bands $(1.2 \mathrm{GHz}, 4.1 \mathrm{GHz}$ and $7.6 \mathrm{GHz})$ around the centre of the line the intensity and shape of this atmospheric water line emission within the directional pattern of the instrument could be derived from the brightness temperature. Then the PWV is calculated by iteration of the water wapour density in the radiative transfer equation. Since September 1998 such a radiometer (two radiometers starting in March 1999) has been used for the long-term site test measurements at Llano de Chajnantor - $5000 \mathrm{~m}$ high plateau in the Atacama Desert region of northern Chile, chosen for the ALMA project (Delgado, 2002).

\section{Summary and conclusions}

The large radio-telescope facility has to be completed in few years on the Suffa Plateau in Uzbekistan. The current view of the building site with parts of the facility created is presented in Fig. 4. Recently the project was considerably updated and modernized to fit present-day requirements for a first-class instrument. The site is very dry and has quite good seeing conditions, both in visual and radio ranges. A new deeper site-testing programme is being created, in order to learn more about the radio astro-climate of the site in more detail, and to prepare the ground for 'radio-weather' forecasts. The last will increase the efficiency of future observations, particularly in $\mathrm{mm}$ waves. We welcome any proposals and will be glad if you join us both in completing the facility complex and exploring the radio-weather. 


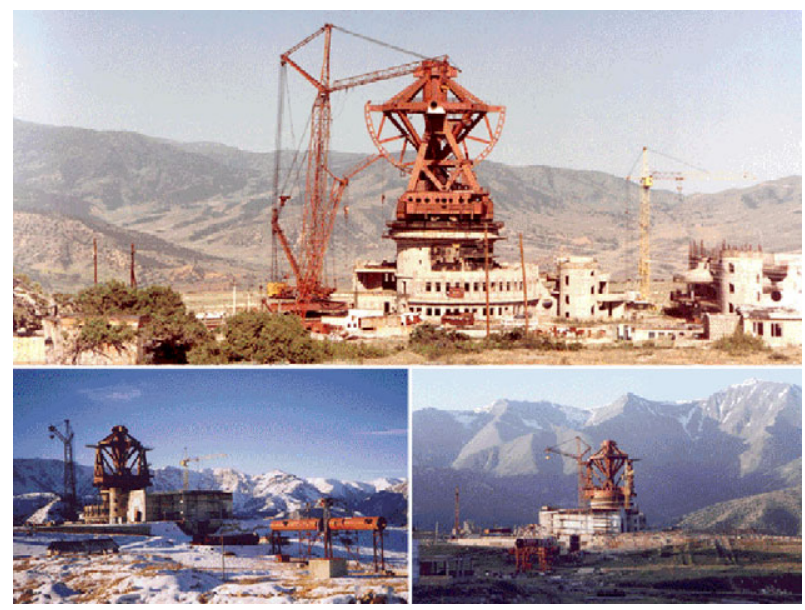

Figure 4. Current panoramic snapshots of creating the Suffa Observatory

\section{Acknowledgements}

We would like to acknowledge our colleagues from ASC/LPI of RAS and other involved organizations who are actively working on the project and promote it. The authors also thank a referee for the useful comments.

\section{References}

Delgado, G. 2002, in: J. Vernin, Z. Benkhaldoun, \& C. Munoz-Tunon (eds.), Astronomical Site Evaluation in the Visible and Radio Range. ASP Conference Proceedings(ISBN: 1-58381106-0. San Francisco, Astronomical Society of the Pacific), Vol. 266, p. 246

Hills, R., Gibson, H., Richer, J., Smith, H., Belitsky, V., Booth, R., Urbain, D. 2001, ALMA Memo 352, pp. 16

Hojaev, A.S., Shanin, G.I. 1996, Journal of Korean Astronomical Society vol. 29, p. S411

Kardashev, N.S. 1992, Suffa International Radio Astronomy Observatory Report in NASA Headquarter, Washington

Kardashev, N.S., Andreyanov, V.V., Gvamichava, A.S., Likhachev, S.F., and Slysh, V.I. 1995, Acta Astronautica vol. 37, p. 271

Shanin, G.I. 1996, Precision Radio Telescope RT70, Abstracts of First Conference on Space Research, Technology and Conversion, Tashkent, p. 27

Trimble, V. 2001, Future - A Year of Discovery: Astronomy Highlights of 2000 Sky and Telescope (February 2001), p. 50 


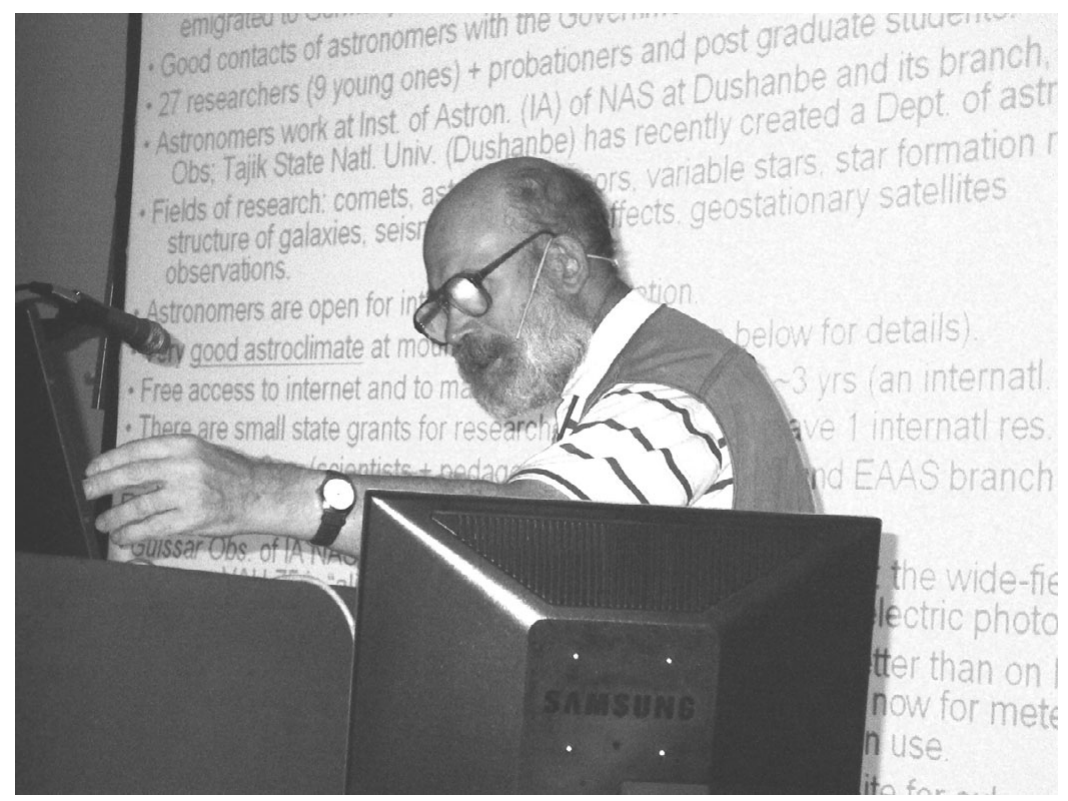

Nikolai Bochkarev

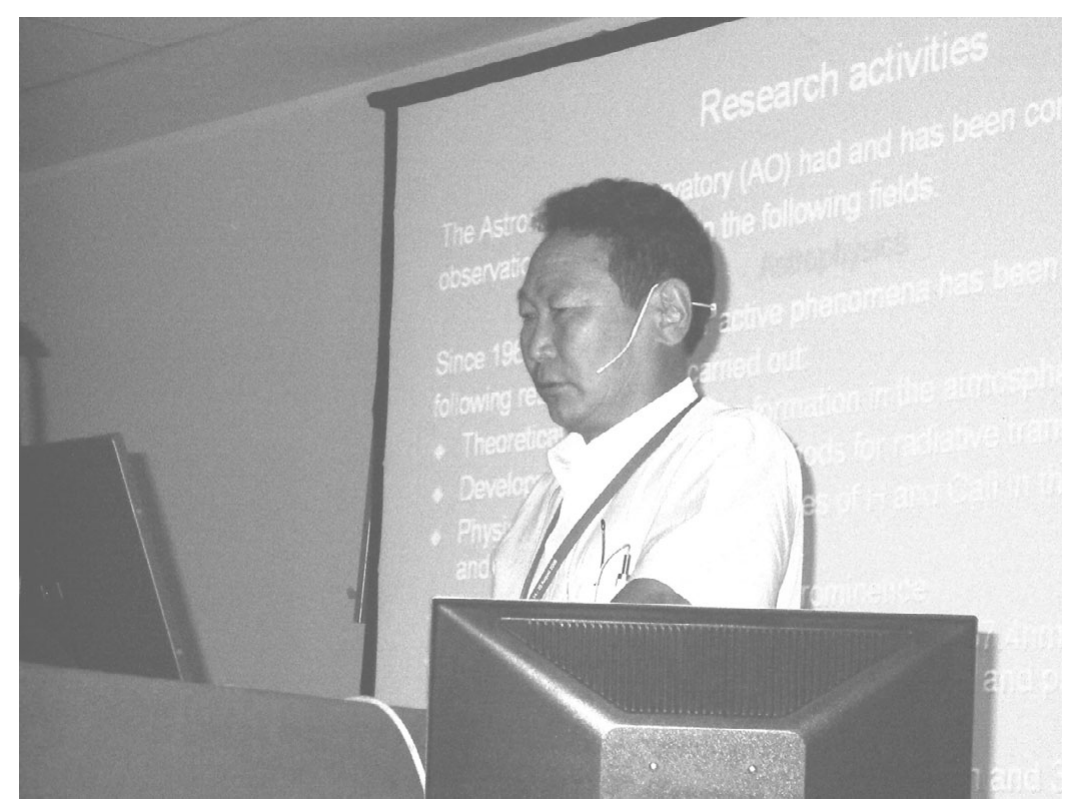

Damdin Batmunkh 